\title{
Essex Rovers Team Description
}

\author{
Huosheng $\mathrm{Hu}$, Dongbing $\mathrm{Gu}$, Bo Li \\ Department of Computer Science, University of Essex \\ Wivenhoe Park, Colchester CO4 3SQ, UK \\ Email: \{hhu,dgu, bli $\} @$ essex.ac.uk
}

\begin{abstract}
This article introduces our current research efforts to build a multi-agent system for cooperation and learning of multiple Sony legged robots in the RoboCup domain. A behaviour-based hierarchy is proposed for our Essex Rovers robot soccer team to achieve intelligent actions in real time, which includes both a neural network based color detection algorithm and a fuzzy logic controller.
\end{abstract}

\section{Introduction}

RoboCup (Robot World Cup Initiative) provides a challenging environment for research in a team of robotic systems that need to achieve concrete objectives, particularly in the presence of an adversary team. In the Sony Legged Robot League [2], there are three Sony legged robots in each team. According to the competition rules, the field size and other teams' experience [9], our team, Essex Rovers, has adopted the team formation with two robots playing as attackers and one as goalie. For the first time to take part in the competition, it was too much to set down for our team. Fortunately we have manuals and support from Sony [8] and the reports from other teams competed in RoboCup99 [1]. All these were really helpful.

Our preparation for the competition can be divided into three stages. At the 1st stage, we read every piece of information available to see how to program and tested each example that Sony provided in order to see how the robot operates. Then we came to the 2nd stage to construct our team strategies. We targeted two goals under the pressure of time limit: to quickly set up everything for the robot to play soccer and to gradually modify the program that appeared not reasonable. This strategy does reduce the stress we faced and it took us about three months to have a fully working team. In the 3rd stage, we refined our sensing and control strategies gradually. Our team showed the abilities to play the match and challenges during the last few days before leaving for Melbourne. Our research is focused on learning and evolving of the Sony legged robots based on the behaviour structure.

\section{Architecture for building the intelligent robot}

We have designed agent-based control architecture for our robots, which consists of three modules: Perception, Cognition, and Action [3]. As shown in figure 1, three modules are placed under the OPEN_R structure and communicate with each other through their connections. The Perception module takes all sensor information as inputs, including images from a CCD camera, ranges from an infrared range finder, postures from gyros, pressures from a head touch sensor. It maintains a local map relative to the robot centre and a global map relative to the left corner of the field by updating the localization of different objects defined in the map based on different sensory information. Both the local map and the global map are $\mathrm{C}++$ objects shared 
through their points. The Cognition and Action modules form hierarchical behaviour structure [4].

The high-level behaviours in the Cognition module behave as the states of a non-linear dynamic process actuated by the two maps, which will be stabilized either in one state such as FINDBALL behaviour or in the limit cycle states formed by different behaviours. The low-level

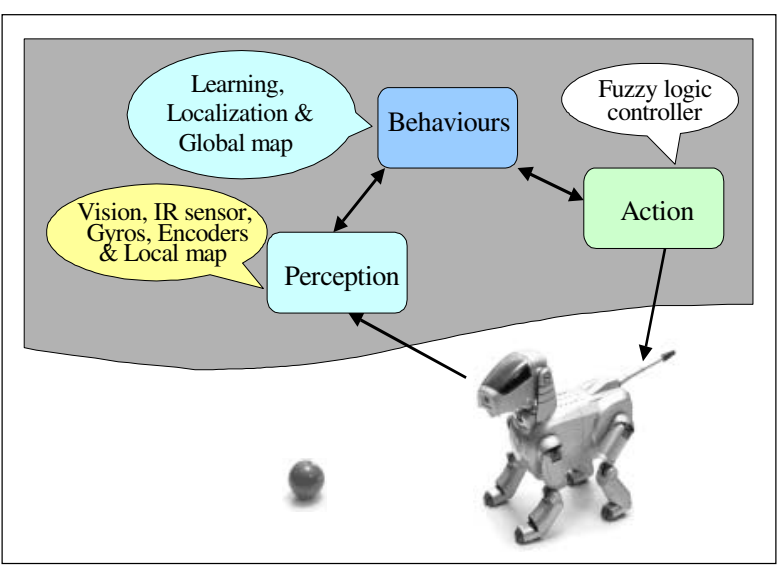

Figure 1 Agent architecture for Essex Rovers behaviours in the Action module are called basic behaviours, each of which can complete a primitive action such as APPROACH_TO_BALL according to the information from the maps. The fuzzy logic controller is used here to implement the action while handling uncertainty [6].

\section{Vision}

We developed a tool called YUVC by using Microsoft Visual C++ to generate the Colour Detection Table (CDT) for image thresholding based on the built-in threshold hardware of the robot. The CDT has 8 tables for 8 colours, each of which has 32 levels of luminance value (Y) for 4 min-max threshold colour values of $U, V$ in the YUV colour space. YUVC is a window-based tool and makes manual colour separation easy by moving mouse only. Furthermore it also provides the ability to simulate the image processing with same $\mathrm{C}++$ code employed in real robots.

\section{Object recognition}

The procedures for object recognition [3] are:

- Image capture - AIBO provides the colour images in YUV space and each pixel in the image is represented by 3 bytes of $\mathrm{Y}, \mathrm{U}$, and $\mathrm{V}$ values. It also provides 8 colour images after each captured image being threshold by hardware. The hardware threshold makes use of $32 \mathrm{Y}$ levels and there are 2 thresholds for $U$ and other 2 thresholds for $\mathrm{V}$ at each level. This will lead to 32 rectangles in $\mathrm{U}$ and $\mathrm{V}$ frames for each colour. In order to select the thresholds for the hardware, we developed a software tool that can manually label the different colour and find the thresholds among the labeled pixels.

- Image segmentation - The threshold image may contain noise due to the luminance condition. We use morphology filters to de-noise the image since the detected object's shape is known a prior [7]. For a binary image, there are two operators normally used in a morphology filter, namely dilation and erosion. Morphologically filtering an image by an opening or closing operation corresponds to the ideal non-realizable band-pass. 
- Image representation - Before object understanding the similar adjacent pixels have to be grouped into the connected regions. This is typically expensive operation that severely impacts real time performance. We calculate the run length encoding (RLE) to represent the image in order to make our next image operation based on RLE not on individual pixels. Region identification can be performed in two passes, see [3] for more details.

\section{Localization}

There are two maps for localization in our team, namely the local map relative to the robot center and the global map relative to the left corner of the field, both of which maintained by the sensory information. The object features are kept in the maps, for example, the distance, view angle, pixel size in the local map and 2-D co-ordinations and orientation in the global map. A certainty value (CV) that is exponentially decayed is assigned to each object in the map. The purpose of $\mathrm{CV}$ is to equip the robots with memory in order for them to make decisions when the desired objects are lost from their sight. For the attackers, the triangulation that is calculated by recognizing three poles is used for localization. The condition for activating the localization is limited in only a few cases, such as losing ball for a certain time, in order to save time to react with the dynamic environment. For the goalie, a set of heuristic rules is developed for localization. The rule set takes the goals, poles, ground, and white lines in both the local map and global map as inputs and is more efficient than triangulation in some situations in which some poles are perhaps obscured by other robots or not recognized due to the changing lighting condition. The localization will be trigged when the robot has no ball or cannot see the ball.

\section{Behaviours}

There are two level behaviours in our robots. The high-level behaviours contained in the Cognition module as the states of a non-linear dynamic process actuated by the two maps, which will be stabilized either in one state such as FINDBALL behaviour or in the limit cycle states formed by different behaviours. The low-level behaviours in the Action module are called basic behaviour, each of which can complete a primitive action such as APPROACH_TO_BALL according to the information from the maps. Both the attackers and the goalie have their different Cognition modules, and these modules then form team behaviours such as localization, co-operation, etc.

A reactive control scheme [3] is employed in the low level for each behaviour with the sensory data from the local map as inputs and moving command from OPEN-R moving modular as outputs. The sensor data from the local map is corrupted with noise, which forces us to use a fuzzy controller to complete the behaviour issued from the Cognition module.

\section{Action/Execution}

After testing for different walking styles provided by MoNet object of Aperios, we use the normal walking command as our team's motion styles. The two custom walking commands, SideWalking and Rolling, are designed by trial and error to make the robot move more flexibly. The kick command of MoNet is not efficient in testing 
due to its slow moving and inaccurate angle. Therefore we use the fast walking style for kicking instead. Head motion for finding the ball is also difficult to control since there is a time delay between the time when the robot finds the ball and the time when the command is executed. The head angles are recorded when the ball is founded and then head is moved back to that position after the current command is finished.

\section{Conclusion}

In this article, we present our effect on training Sony legged robots for the competition in RoboCup-2000. The vision processing and the behaviour construction are two key aspects being focused by our team. A morphology filter is employed for image pre-processing. The behaviours serve as basic building blocks for our modular software system. The structure of two level behaviours can de-composite the task into contextually meaningful units that couple perception and action tightly. Fuzzy logic implementation further enhances the abilities of the system in face of the uncertain situation [10].

Based on the current system, we will focus on the robot's learning and evolving abilities for the next competition, particularly on the vision system and cooperative behaviours. The main shortcomings for our team in RoboCup-2000 are the slow motion and inefficient kicking. We will try to modify the walking style to improve its mobility and accuracy, and add effective kicking behaviours like the UNSW team.

Acknowledgements: We would like to thank Masahiro Fujita and other staff members in the Digital Creatures Laboratory, Sony Corporation, Japan for their technical support. This research is financially supported by the Royal Society Research Grant 574006.G503 and the Essex University Research Promotion Fund DDPB40.

\section{References:}

1. M. Asada, et al, RoboCup: Today and tomorrow what we have learned, Artificial Intelligent, 110, pages 275-292, 1999.

2. M. Fujita, H. Kitano, K. Kageyama, A reconfigurable robot platform, Int. Journal of Robotics and Autonomous Systems, Vol. 29, No. 2, pages 119-132, 1999.

3. D. Gu and H. Hu, Towards Learning and Evolving of a Team of Sony Legged Robots, The Workshop on Recent Advances on Mobile Robots, Leicester, 29 June 2000

4. $\mathrm{H}$. Hu and D. Gu, A Multi-Agent System for Cooperative Quadruped Walking Robots, IASTED Int. Conf. Robotics and Applications, pages 182-186, Honolulu, Hawaii, USA, August 2000

5. H. Kitano, et al, Sony Legged Robot for RoboCup Challenge, Proc. of the 1998 IEEE Int. Conf. on Robotics and Automation, Leuven, Belgium, pages 2605-2612, May 1998.

6. A. Saffiotti, E. Ruspini, and K. Konolige, Using Fuzzy Logic for Mobile Robot Control, Practical Applications of Fuzzy Technologies, In H-J. Zimmermann, editor, Kluwer Academic Publisher, pages 185-206, 1999.

7. M. Sonka, V. Hiavac, and R. Boyle, Image Processing, Analysis, and Machine Vision, PWS Publishing, 1998.

8. Sony Corporation, Entertainment Robot ERS-110: Operation Manual, 1999

9. M. Veloso, E. Pagello, H. Kitano (Eds.), Lecture Notes in Artificial Intelligence, 1856, RoboCup-99: Robot Soccer World Cup III, Springer, 2000. 